\title{
Comparação de bulas de duas marcas de tiras reagentes utilizadas no exame químico de urina
}

\section{Comparison of product labelings of two marks of reagent strips for the chemical examination of urine}

Adriana Scotti da Silva Colombeli1; Miriam Falkenberg2

\section{unitermos resumo}

Urinálise

Tiras reagentes

Exame quimico

Comparação de bulas

Introdução: O exame de urina proporciona informações sobre patologias renais e do trato urinário, bem como algumas moléstias extra-renais. Usualmente o exame químico de urina é feito com tiras reagentes, objetivando tornar a determinação mais rápida, simples e econômica. Objetivos: Comparar bulas de duas marcas de tiras amplamente utilizadas em laboratórios de urinálise (Roche Combur ${ }^{10}$ Test $^{\circledR}$ UX e Bayer Multistix ${ }^{\circledR} 10$ SG). Material e método: Compararam-se as bulas quanto aos princípios utilizados nas determinações de $\mathrm{pH}$, proteínas, glicose, cetonas, hemoglobina, bilirrubina, urobilinogênio, nitrito, densidade e leucócitos, além das informações sobre possíveis interferências. Resultados: Foram verificadas diferenças nos reagentes utilizados para detecção dos parâmetros, como é o caso do urobilinogênio (a tira Multistix usa o reagente de Ehrlich, menos específico e mais propenso a interferências analíticas que o sal de diazônio derivado de metoxibenzeno, utilizado na tira Roche); para nitrito, proteína, glicose, bilirrubina e hemoglobina as diferenças foram mais sutis. Discussão: Detectou-se diversidade de informações quanto a possíveis interferentes, o que talvez possa ser justificado parcialmente pelas diferenças nos reagentes. Também foram verificadas diferenças nas informações sobre interferências de um idioma para outro, destacando-se a omissão de algumas delas na bula em português. Observou-se grande disparidade na avaliação da intensidade da reação e sua expressão em cruzes, como, por exemplo, no parâmetro glicose, o que pode levar a erros na interpretação do laudo laboratorial. Conclusão: As observações registradas reforçam a importância de padronizações no exame parcial de urina.
\end{abstract}

Background: The urinalysis provides information about renal and urinary diseases, as well as about some extra renal diseases. The chemical examination of urine is done with reagent strips, which allows a quick, simple and economic analyze. Objectives: To compare drug labelings of two marks of reagent strips in urinalysis (Roche Combur $^{10}$ Test $^{\circledR}$ UX and Bayer Multistix ${ }^{\circledast} 10$ SG). Material and Method: The principles of $p H$, protein, glucose, ketone bodies, hemoglobin, bilirubin, urobilinogen, nitrite, specific gravity and leukocytes determinations, as well as the information about possible interferences were compared. Results: There were differences in the detection's reagents of some parameters, like urobilinogen (The Ehrlich's reagent present in Multistix is less specific and more propense to analytical interferences than diazonium salt derived of methoxybenzene, used by Roche strip); the differences in the reagents used for nitrite, protein, glucose, bilirubin and hemoglobin determinations were relatively subtle. Discussion: There was diversity of information about the potential interferences, which could be justified (at least partially) by differences in the reagents. Differences in the information about interferences had been detected between Spanish and Portuguese versions in the same product labeling, like the omission of some interferences in the product labeling in Portuguese. Great disparity in the reaction's intensity and its expression in crosses were observed (e.g. for glucose), and it could lead to misunderstanding in the interpretation of laboratorial findings. Conclusion: These data reinforce the importance of standardizations in the urinalysis.

1. Aluna de mestrado do Programa de Pós-graduação em Farmácia da Universidade Federal de Santa Catarina (UFSC); bioquímica do Hospital Universitário, do Centro de Ciências da Saúde (CCS) da UFSC.

2. Professora do Departamento de Ciências Farmacêuticas do CCS/UFSC. 


\section{Introdução}

A análise da urina foi o começo da medicina laboratorial. Referências ao estudo da urina foram encontradas em desenhos dos homens das cavernas e nos hieróglifos egípcios, como o papiro cirúrgico de Edwin Smith, cujos quadros representavam os médicos da Antigüidade examinando um frasco de urina. Embora não contassem com métodos sofisticados de exame, eles eram capazes de obter informações diagnósticas de observações básicas, como cor, turvação, odor, volume, viscosidade e até mesmo a presença de açúcar em certas amostras, por observar a aproximação de formigas e outros insetos da urina de alguns pacientes ${ }^{(3,14)}$. Muitos nomes conhecidos da medicina estão ligados ao estudo da urina, inclusive Hipócrates (460-370 a.C.), que escreveu sobre uroscopia. Em decorrência do aumento do conhecimento científico-tecnológico observado no século XX, a realização do exame de urina evoluiu, tornando-se a ciência plena, denominada urinálise ${ }^{(14)}$.

O exame de urina proporciona ao clínico informações precisas sobre patologias renais e do trato urinário, bem como sobre algumas moléstias extra-renais. Além disso, permite uma avaliação da função renal e fornece indícios sobre a etiologia da disfunção. Por sua simplicidade, baixo custo e facilidade na obtenção da amostra para análise, é considerado exame de rotina ${ }^{(6,7,10)}$.

Usualmente a análise dos constituintes bioquímicos da urina é feita através de tiras reagentes, objetivando tornar a determinação de elementos da urina mais rápida, mais simples e mais econômica ${ }^{(7,16)}$. Atualmente há no mercado instrumentos que executam a leitura das fitas reagentes, melhorando assim o grau de precisão ao eliminar parte do elemento subjetivo inerente à leitura das mudanças de cor pelo olho humano ${ }^{(10)}$. As tiras reativas de urina constituem um meio simples e rápido de realizar dez ou mais análises bioquímicas clinicamente importantes, como $\mathrm{pH}$, proteínas, glicose, cetonas, hemoglobina, bilirrubina, urobilinogênio, nitrito, densidade e leucócitos ${ }^{(14)}$.

Exames laboratoriais solicitados em caso de suspeita de infecção do trato urinário envolvem, por exemplo, a detecção de bactérias e/ou leucócitos por tiras reagentes de urina, exame microscópico do sedimento ou cultura de urina. O desenvolvimento de cor na área de esterase leucocitária indica a presença de leucócitos na urina, presumivelmente atraídos pela invasão de microorganismos no trato urinário. Uma coloração na área reagente de nitrito sugere a presença de bactérias, capazes de reduzir nitrato a nitrito. Lyon et al. ${ }^{(8)}$ determinaram em $58 \%$ a sensibilidade da esterase de leucócitos em prever a quantidade de leucócitos no sedimento urinário, mas também observam uma correlação negativa, estatisticamente significativa, entre a densidade e os resultados na esterase de leucócitos ${ }^{(8)}$. Esta correlação explicaria, ao menos em parte, a sensibilidade relativamente baixa no teste de esterase de leucócitos. Segundo Sultana et al. ${ }^{(15)}$, tiras reagentes de urina fornecem informações que melhoram significativamente a precisão no diagnóstico de infecção do trato urinário.

\section{Objetivo}

O objetivo deste trabalho foi analisar comparativamente duas das marcas mais conhecidas de tiras entre as atualmente utilizadas em laboratórios de urinálise (Roche Combur ${ }^{10}$ Test $^{\circledR}$ UX e Bayer Multistix ${ }^{\circledR} 10$ SG) quanto às informações referentes ao princípio do teste utilizado, bem como outras relativas aos parâmetros avaliados, com destaque para as potenciais interferências.

\section{Material e método}

Foram comparadas as bulas das tiras Roche Combur ${ }^{10}$ Test $^{\circledR}$ UX e Bayer Multistix ${ }^{\circledast} 10$ SG quanto aos princípios utilizados nas determinações urinárias de $\mathrm{pH}$, proteínas, glicose, cetonas, sangue, bilirrubina, urobilinogênio, nitrito, densidade e leucócitos, além das informações quanto ao limite mínimo de detecção, intervalos de leitura e possíveis interferências nos parâmetros avaliados.

\section{Resultados}

As bulas das marcas Combur e Multistix apresentam diferentes idiomas disponíveis, sendo inglês, português, sueco e dinamarquês na tira Combur e somente português e espanhol na marca Multistix.

A Tabela 1 apresenta os reagentes empregados que se relacionam com o princípio de detecção no qual se baseiam as análises. A sensibilidade e/ou limite mínimo declarado para cada parâmetro, bem como seus intervalos de leitura, são apresentados nas Tabelas $\mathbf{2}$ e 3, respectivamente.

As potenciais interferências relatadas nas bulas para cada parâmetro são especificadas a seguir.

\section{Bilirrubina}

A bula da tira Combur cita que "outras substâncias contidas na urina provocam coloração amarela mais ou menos 


\begin{tabular}{|c|c|c|}
\hline Parâmetro/tira & Combur $^{10}$ Test $^{6}$ UX & Multistix 10 SG \\
\hline Bilirrubina & $\begin{array}{l}\text { Sal de diazônio diclorobenzeno } \\
16,7 \mu \mathrm{g}\end{array}$ & $\begin{array}{l}\text { 0,4\% } \mathrm{p} / \mathrm{p} \text { de sal de diazônio 2,4-dicloroanilina; } 37,3 \% \\
\mathrm{p} / \mathrm{p} \text { de solução tampão; } 62,3 \% \mathrm{p} / \mathrm{p} \text { de componentes } \\
\text { não-reativos }\end{array}$ \\
\hline Cetona & $\begin{array}{l}\text { Nitroprussiato de sódio 157,2 } \mu \mathrm{g} ; \\
\text { glicina } 4,2 \mathrm{mg}\end{array}$ & $\begin{array}{l}\text { 7,1\% } \mathrm{p} / \mathrm{p} \text { de nitroprussiato de sódio; } 92,9 \% \mathrm{p} / \mathrm{p} \text { de } \\
\text { solução tampão }\end{array}$ \\
\hline Densidade & $\begin{array}{l}\text { Azul de bromotimol } 36 \mu \mathrm{g} \text {; ácido } \\
\text { etilenoglicoldiaminoetiletertetracético } \\
182,8 \mu \mathrm{g}\end{array}$ & $\begin{array}{l}2,8 \mathrm{p} / \mathrm{p} \text { de azul de bromotimol; } 68,8 \% \mathrm{p} / \mathrm{p} \text { de polímero } \\
\text { (metil-vinil-éter-maléico anidro); } 28,4 \% \mathrm{p} / \mathrm{p} \text { de } \\
\text { hidróxido de sódio }\end{array}$ \\
\hline Glicose & $\begin{array}{l}\text { Tetrametilbenzidina } 103,5 \mu \mathrm{g} \text {; Glicose- } \\
\text { oxidase } 6 \mathrm{U} \text {, Peroxidase } 35 \mathrm{U}\end{array}$ & $\begin{array}{l}2,2 \% \mathrm{p} / \mathrm{p} \text { de glicose-oxidase (microbiana, } 1,3 \mathrm{UI}) ; 1 \% \\
\mathrm{p} / \mathrm{p} \text { de peroxidase ( } 3.300 \mathrm{UI}) ; 8,1 \% \mathrm{p} / \mathrm{p} \text { de iodeto de } \\
\text { potássio; } 69,8 \% \mathrm{p} / \mathrm{p} \text { de solução tampão; } 18,9 \% \mathrm{p} / \mathrm{p} \text { de } \\
\text { componentes não-reativos }\end{array}$ \\
\hline Hemoglobina & $\begin{array}{l}\text { Tetrametilbenzidina } 52,8 \mu \mathrm{g} ; \\
\text { dimetildiidroperoxiexano } 297,2 \mu \mathrm{g}\end{array}$ & $\begin{array}{l}6,8 \% \mathrm{p} / \mathrm{p} \text { de diidroperóxido de diisopropilbenzeno; } 4 \% \\
\mathrm{p} / \mathrm{p} \text { de } 3,3^{\prime}-5,5^{\prime} \text {-tetrametilbenzidina; } 48 \% \mathrm{p} / \mathrm{p} \text { de solução } \\
\text { tampão; } 41,2 \% \mathrm{p} / \mathrm{p} \text { de componentes não-reativos }\end{array}$ \\
\hline Leucócitos & $\begin{array}{l}\text { Éster de indoxil } 15,5 \mu \mathrm{g} ; \text { sal de } \\
\text { diazônio metoxi-morfolino-benzeno } \\
5,5 \mu \mathrm{g}\end{array}$ & $\begin{array}{l}0,4 \% \mathrm{p} / \mathrm{p} \text { de éster derivado de aminoácido pirrólico; } 0,2 \% \\
\mathrm{p} / \mathrm{p} \text { de sal de diazônio; } 40,9 \% \mathrm{p} / \mathrm{p} \text { de solução tampão; } \\
58,5 \% \mathrm{p} / \mathrm{p} \text { de componentes não-reativos }\end{array}$ \\
\hline Nitrito & $\begin{array}{l}\text { Hidroxitetraidrobenzoquinoleína } \\
33,5 \mu \mathrm{g} \text {; sulfanilamida } 29,1 \mu \mathrm{g}\end{array}$ & $\begin{array}{l}\text { 1,4\% } \mathrm{p} / \mathrm{p} \text { de ácido } \mathrm{p} \text {-arsanílico; } 1,3 \% \mathrm{p} / \mathrm{p} \text { de } 1,2,3,4- \\
\text { tetraidrobenzeno(h)-quinolin-3-ol; } 10,8 \% \mathrm{p} / \mathrm{p} \text { de solução } \\
\text { tampão; } 86,5 \% \mathrm{p} / \mathrm{p} \text { de componentes não-reativos }\end{array}$ \\
\hline $\mathrm{pH}$ & $\begin{array}{l}\text { Azul de bromotimol } 13,9 \mu \mathrm{g} \text {; vermelho } \\
\text { de metila } 1,2 \mu \mathrm{g} ; \text { fenolftaleína } 8,6 \mu \mathrm{g}\end{array}$ & $\begin{array}{l}0,2 \% \mathrm{p} / \mathrm{p} \text { de vermelho de metila; } 2,8 \% \mathrm{p} / \mathrm{p} \text { de azul de } \\
\text { bromotimol; } 97 \% \mathrm{p} / \mathrm{p} \text { de componentes não-reativos }\end{array}$ \\
\hline Proteína & $\begin{array}{l}\text { Tetraclorofenoltetrabromossulfoftaleína } \\
13,9 \mu \mathrm{g}\end{array}$ & $\begin{array}{l}0,3 \% \mathrm{p} / \mathrm{p} \text { de azul de tetrabromofenol; } 97,3 \% \mathrm{p} / \mathrm{p} \text { de } \\
\text { solução tampão; } 2,4 \% \mathrm{p} / \mathrm{p} \text { de componentes não-reativos }\end{array}$ \\
\hline Urobilinogênio & Sal de diazônio metoxibenzeno $67,7 \mu \mathrm{g}$ & $\begin{array}{l}0,2 \% \mathrm{p} / \mathrm{p} \text { de } \mathrm{p} \text {-dietilaminobenzaldeído; } 99,8 \% \mathrm{p} / \mathrm{p} \text { de } \\
\text { componentes não-reativos }\end{array}$ \\
\hline
\end{tabular}

p/p: relação peso/peso.

Tabela 2 Sensibilidade/limite minimo de detecção declarado nas bulas

\begin{tabular}{|c|c|c|}
\hline Parâmetro/tira & Combur $^{10}$ Test $^{6} \mathrm{UX}$ & Multistix 10 SG \\
\hline Bilirrubina & $0,5 \mathrm{mg} / \mathrm{dl}$ & $0,4-0,8 \mathrm{mg} / \mathrm{dl}$ \\
\hline Cetona & $5 \mathrm{mg} / \mathrm{dl}$ & $5-10 \mathrm{mg} / \mathrm{dl}$ \\
\hline Densidade & - & - \\
\hline Glicose & $40 \mathrm{mg} / \mathrm{dl}$ & $75-125 \mathrm{mg} / \mathrm{dl}$ \\
\hline Hemoglobina & $\begin{array}{l}\text { Eritrócitos intactos: } 5 \mathrm{eri} / \mu \mathrm{l} \\
\text { Hemoglobina ou eritrócitos hemolisados: correspondendo a } 10 \mathrm{eri} / \mu \mathrm{l}\end{array}$ & $0,015-0,062 \mathrm{mg} / \mathrm{dl}$ \\
\hline Leucócitos & 10-25 leucócitos/ $/ \mu l$ & 5-15 leucócitos/campo \\
\hline Nitrito & $0,05 \mathrm{mg} / \mathrm{dl}$ & $0,06-0,1 \mathrm{mg} / \mathrm{dl}$ \\
\hline $\mathrm{pH}$ & - & - \\
\hline Proteína & $6 \mathrm{mg} / \mathrm{dl}$ & $15-30 \mathrm{mg} / \mathrm{dl}$ \\
\hline Urobilinogênio & $0,4 \mathrm{mg} / \mathrm{dl}$ & $0,2 \mathrm{mg} / \mathrm{dl}$ \\
\hline
\end{tabular}




\section{Tabela 3 Intervalos de leitura descritos nas bulas para os diferentes parâmetros}

\begin{tabular}{|c|c|c|}
\hline Parâmetro/tira & Combur $^{10}$ Test $^{\circledR}$ UX & Multistix 10 SG \\
\hline Bilirrubina & $\begin{array}{c}\text { Negativo } \\
\text { Positivo (+) } \\
\text { Positivo (++) } \\
\text { Positivo (+++) }\end{array}$ & $\begin{array}{c}\text { Negativo } \\
\text { Positivo (+) baixo } \\
\text { Positivo (++) moderado } \\
\text { Positivo (+++) alto }\end{array}$ \\
\hline Cetona & $\begin{array}{c}\text { Negativo } \\
\text { Positivo }(+) \\
\text { Positivo }(++) \\
\text { Positivo }(+++)\end{array}$ & $\begin{array}{c}\text { Negativo } \\
\text { Traços }-5 \mathrm{mg} / \mathrm{dl} \\
\text { Baixo }-15 \mathrm{mg} / \mathrm{dl} \\
\text { Moderado }-40 \mathrm{mg} / \mathrm{dl} \\
\text { Alto }-80-160 \mathrm{mg} / \mathrm{dl}\end{array}$ \\
\hline Densidade & $1-1,03$ & $1-1,03$ \\
\hline Glicose & $\begin{array}{c}\text { Negativo } \\
\text { Positivo }(+)-50 \mathrm{mg} / \mathrm{dl} \\
\text { Positivo }(++)-100 \mathrm{mg} / \mathrm{dl} \\
\text { Positivo }(+++)-300 \mathrm{mg} / \mathrm{dl} \\
\text { Positivo }(++++)-1.000 \mathrm{mg} / \mathrm{dl}\end{array}$ & $\begin{array}{c}\text { Negativo } \\
\text { Traços }-100 \mathrm{mg} / \mathrm{dl} \\
\text { Positivo }(+)-250 \mathrm{mg} / \mathrm{dl} \\
\text { Positivo }(++)-500 \mathrm{mg} / \mathrm{dl} \\
\text { Positivo }(+++)-1.000 \mathrm{mg} / \mathrm{dl} \\
\text { Positivo }(++++)-\geq 2.000 \mathrm{mg} / \mathrm{dl}\end{array}$ \\
\hline Hemoglobina & $\begin{array}{c}\text { Negativo } \\
\text { Positivo (+) - 5-10 erit/ } \mathrm{ll} \\
\text { Positivo (++) - } 25 \mathrm{erit} / \mu \mathrm{l} \\
\text { Positivo (+++) - } 50 \mathrm{erit} / \mu \mathrm{l} \\
\text { Positivo (++++) - } 250 \mathrm{erit} / \mu \mathrm{l}\end{array}$ & $\begin{array}{c}\text { Negativo } \\
\text { Traços } \\
\text { Positivo (+) baixo } \\
\text { Positivo }(++) \text { moderado } \\
\text { Positivo (+++) alto }\end{array}$ \\
\hline Leucócitos & $\begin{array}{c}\text { Negativo } \\
\text { Positivo }(+)-10-25 \text { leuco } / \mu l \\
\text { Positivo }(++)-75 \text { leuco } / \mu \mathrm{L} \\
\text { Positivo }(+++)-500 \text { leuco } / \mu \mathrm{l}\end{array}$ & $\begin{array}{c}\text { Negativo } \\
\text { Traços } \\
\text { Positivo (+) baixo } \\
\text { Positivo (++) moderado } \\
\text { Positivo (+++) alto }\end{array}$ \\
\hline Nitrito & Negativo/positivo & Negativo/positivo \\
\hline $\mathrm{pH}$ & $5-9$ & 5-8,5 (manual), 5-9 (instrumental) \\
\hline Proteína & $\begin{array}{c}\text { Negativo } \\
\text { Positivo }(+)-30 \mathrm{mg} / \mathrm{dl} \\
\text { Positivo }(++)-100 \mathrm{mg} / \mathrm{dl} \\
\text { Positivo (+++) }-500 \mathrm{mg} / \mathrm{dl}\end{array}$ & $\begin{array}{c}\text { Negativo } \\
\text { Traços } \\
\text { Positivo }(+)-30 \mathrm{mg} / \mathrm{dl} \\
\text { Positivo }(++)-100 \mathrm{mg} / \mathrm{dl} \\
\text { Positivo }(+++)-300 \mathrm{mg} / \mathrm{dl} \\
\text { Positivo }(++++)-\geq 2.000 \mathrm{mg} / \mathrm{dl}\end{array}$ \\
\hline Urobilinogênio & $\begin{array}{c}\text { Normal } \\
\text { Positivo }(+)-1 \mathrm{mg} / \mathrm{dl} \\
\text { Positivo }(++)-4 \mathrm{mg} / \mathrm{dl} \\
\text { Positivo (+++) }-8 \mathrm{mg} / \mathrm{dl} \\
\text { Positivo (++++) }-12 \mathrm{mg} / \mathrm{dl}\end{array}$ & $\begin{array}{l}\text { Normal }-0,2-1 \mathrm{mg} / \mathrm{dl} \\
2 \mathrm{mg} / \mathrm{dl} \\
4 \mathrm{mg} / \mathrm{dl} \\
8 \mathrm{mg} / \mathrm{dl}\end{array}$ \\
\hline
\end{tabular}

intensa", porém não especifica quais seriam tais substâncias. Segundo a bula da Multistix, o indicador (sulfato de indoxila) é capaz de produzir respostas em cores desde o amarelo-alaranjado até o vermelho, que podem interferir na interpretação de leituras positivas ou negativas; metabólitos do etodolaco podem causar resultados falso-positivos ou atípicos. Cores atípicas podem indicar anormalidades do pigmento da bile. 


\section{Cetona}

De acordo com a marca Combur, fenilcetonas e derivados da ftaleína provocam uma coloração vermelha na zona de teste que, no entanto, se distingue nitidamente da coloração violeta produzida pelos corpos cetônicos. Captopril, mesna (sal sódico do ácido 2-mercaptoetanolsulfônico) e outras substâncias contendo grupos sulfidrila podem originar resultados falso-positivos. Nas tiras Multistix, falsos resultados de traços podem ocorrer com amostras de urinas altamente pigmentadas ou contendo altas concentrações de metabólitos do levodopa.

\section{Densidade}

Ambas as bulas informam a necessidade de adicionar 0,005 ao valor da densidade em urinas em determinado $\mathrm{pH}$ ( $\geq 7$ para a tira Combur $e \geq 6,5$ para a Multistix). Em leituras instrumentais essa correção é feita automaticamente. A presença de proteína pode causar leituras elevadas (100 a 500 mg/dl, Combur; 100 a 750 mg/dl, Multistix). A bula da marca Combur afirma que em casos de cetoacidose a densidade lida tende a ser elevada. Urinas alcalinas altamente tamponadas podem causar baixas leituras, segundo a bula da tira Multistix.

\section{Glicose}

Nas tiras Combur o teste independe do $\mathrm{pH}$ e da densidade da urina, e não é afetado pela presença de corpos cetônicos. A influência do ácido ascórbico é consideravelmente eliminada. Em concentrações de glicose $\geq 100 \mathrm{mg} / \mathrm{dl}$ $(5,5 \mathrm{mmol} / \mathrm{l})$, mesmo havendo concentrações elevadas de ácido ascórbico, resultados falso-negativos são improváveis. Ao contrário, nas tiras Multistix, altos níveis de cetona ( $\geq 4 \mathrm{mmol} / \mathrm{l}$ ou $40 \mathrm{mg} / \mathrm{dl}$ ) podem causar falso-negativos para amostras contendo pequenas quantidades de glicose (4-7 mmol/l ou 75-125 mg/dl).

\section{Hemoglobina}

Segundo a bula da Combur, após atividade física, como, por exemplo, jogging intenso, podem surgir valores elevados de eritrócitos e proteínas, sem que isso seja sinal de doença; o ácido ascórbico quase não tem influência no resultado do teste. De acordo com a bula da marca Multistix, o captopril pode reduzir a sensibilidade, e certos contaminantes oxidantes, como o hipoclorito, podem produzir resultados falso-positivos, enquanto a peroxidase microbiana associada à infecção do trato urinário pode causar reação falso-positiva.

\section{Leucócitos}

A bula Combur afirma que a reação não é afetada pela presença de bactérias, Trichomonas ou eritrócitos na urina. O formaldeído (estabilizador) e a antibioticoterapia à base de imipeném, meropeném ou ácido clavulânico podem provocar reações falso-positivas. Em urinas com cor intrínseca intensa (por exemplo, devido à presença de bilirrubina ou nitrofurantoína), a coloração da reação pode ser mascarada. Excreções urinárias de proteína superiores a $500 \mathrm{mg} / \mathrm{dl}$ podem diminuir a intensidade da coloração da reação, assim como elevadas doses diárias de cefalexina ou gentamicina. A cefalexina também é citada pela bula da tira Multistix, além de cefalotina ou altas concentrações de ácido oxálico como causadores da diminuição dos resultados dos testes. Concentrações elevadas de glicose ( $3 \mathrm{~g} / \mathrm{dl}$, Multistix; $2 \mathrm{~g} / \mathrm{dl}$, Combur) podem diminuir a intensidade da coloração. A tetraciclina, segundo a marca Multistix, pode diminuir a reatividade e altos níveis do fármaco podem causar uma reação falso-negativa.

\section{Nitrito}

Segundo a bula da marca Multistix, resultados falso-negativos podem ocorrer com a incubação por curto período na bexiga, na ausência de nitrato na dieta ou na presença de microorganismos patológicos não-reativos. Para a tira Combur, nada é relatado.

\section{pH}

Não houve relatos de interferências na determinação de $\mathrm{pH}$.

\section{Proteína}

$\mathrm{Na}$ bula da tira Combur consta que quinina, quinidina, cloroquina, tolbutamida e pH elevado (até 9) não exercem influência sobre o teste. Podem-se obter resultados falsamente positivos após infusões com polivinilpirrolidona ou devido à presença de resíduos de desinfetantes com amônio quaternário ou cloroexidina no recipiente de coleta da urina. Nenhum relato a respeito é citado pela bula do produto Multistix.

\section{Urobilinogênio}

Segundo o fabricante da marca Combur, o teste é específico para urobilinogênio e não está sujeito às interferências conhecidas da prova de Ehrlich. Grandes quantidades de bilirrubina coram a zona de teste momentaneamente de amarelo e podem, passados cerca de 60 segundos, produzir 
coloração verde a azul. A tira Multistix utiliza o reagente de Ehrlich, portanto essa área de teste pode reagir com substâncias interferentes conhecidamente reativas com esse reagente, tais como ácido $p$-aminossalicílico e sulfonamidas. Reações atípicas podem ser obtidas na presença de altas concentrações de ácido $p$-aminobenzóico e resultados falso-negativos, na presença de formalina. A reatividade da tira aumenta com a temperatura, a qual é ótima entre $22^{\circ} \mathrm{C}$ e $26^{\circ} \mathrm{C}$.

\section{Discussão}

\section{Princípios das reações de detecção}

Comparando-se as bulas das tiras reagentes estudadas, verificamos diferenças nos reagentes utilizados para detecção dos parâmetros (Tabela 1).

\section{Bilirrubina}

As tiras reagentes para detecção de bilirrubinúria geralmente baseiam-se na reação da bilirrubina com um sal de diazônio derivado de 2,4-dicloro-anilina ou 2,6-diclorobenzeno em meio ácido, produzindo cores que variam do bronze ou rosado ao violeta ${ }^{(2,13)}$. A Multistix utiliza o sal de diazônio do tipo citado primeiramente, enquanto a tira da Roche emprega um derivado de diclorobenzeno não claramente especificado como derivado do 2,6-diclorobenzeno.

\section{Cetona}

Para detecção de cetona (ácido acetoacético), ambas as tiras utilizam como reagente o nitroprussiato de sódio, que reage com o ácido acetoacético em meio alcalino, produzindo cor púrpura. A glicina é citada como reagente integrante da tira da Combur, possivelmente como parte do tampão.

\section{Densidade}

A avaliação da densidade baseia-se numa alteração na constante de dissociação (pK) do polieletrólito, que se ioniza proporcionalmente à quantidade de íons em solução, produzindo íons hidrogênio que provocam uma queda no $\mathrm{pH}$, detectada pelo indicador azul de bromotimol. À medida que a densidade aumenta, o azul de bromotimol muda do verde-azulado para verde, e finalmente para amarelo-esverdeado ${ }^{(14)}$. Ambas as tiras utilizam azul de bromotimol como indicador, porém diferentes polieletrólitos.

\section{Glicose}

A detecção de glicose é feita através de uma mistura de glicose-oxidase, peroxidase, cromogênio e tampão para produzir uma reação enzimática seqüencial dupla. Na primeira etapa, a glicose-oxidase catalisa a reação entre a glicose e o ar do meio ambiente para produzir ácido glicônico e peróxido. Em seguida, a peroxidase catalisa a reação entre o peróxido e o cromogênio para formar um complexo oxidado colorido que revela a presença de glicose ${ }^{(11,14)}$. Os reagentes empregados como cromógenos diferem nas tiras analisadas: iodeto de potássio (Multistix) e tetrametilbenzidina (Roche).

\section{Hemoglobina}

As análises químicas para detecção de hemoglobina utilizam a atividade da pseudoperoxidase da hemoglobina para catalisar uma reação entre o peróxido de hidrogênio e o cromogênio (tetrametilbenzidina), produzindo cromogênio oxidado, de coloração azul(14). As tiras analisadas utilizam como hidroperóxidos o dimetil-diidroperóxi-hexano (Roche) ou o diidroperóxido de diisopropilbenzeno (Multistix).

\section{Leucócitos}

Na detecção de leucócitos ocorre uma reação química enzimática que utiliza as esterases presentes nos granulócitos para hidrolisar o éster do ácido indoxilcarbônico e produzir indoxila, que reage com o sal de diazônio e dá origem à cor púrpura ${ }^{(14)}$.

\section{Nitrito}

O fundamento bioquímico da prova do nitrito é a capacidade que certas bactérias têm de reduzir o nitrato, constituinte normal da urina, e convertê-lo em nitrito, que normalmente não aparece na urina. Em pH ácido, os nitritos presentes reagem com uma amina aromática (ácido $p$-arsanílico ou sulfanilamida), formando um sal de diazônio que em seguida reage com 3-hidróxi-1,2,3,4-tetraidrobenzil(H)-quinolina e produz coloração rosa ${ }^{(13)}$. A marca Multistix utiliza como amina aromática o ácido $p$-arsanílico e a tira Roche, a sulfanilamida.

$\mathrm{pH}$

As tiras Roche Combur ${ }^{10}$ Test $^{\circledR}$ UX e Bayer Multistix ${ }^{\circledR}$ 10 SG medem o pH urinário em variações de unidade entre 5 e 9 , usando um sistema de indicador duplo do vermelho de metila e o azul de bromotimol. O vermelho de metila atua como indicador na faixa de 4,4 a 6, produzindo uma 
mudança do vermelho para o amarelo; o azul de bromotimol passa de amarelo para azul e a faixa de ação situa-se entre 5,8 e 7,4. A tira da Roche inclui um terceiro indicador, a fenolftaleína, que é incolor até o pH 8,2 e adquire cor vermelha em meio alcalino até o $\mathrm{pH} 10$, acima do qual torna-se novamente incolor ${ }^{(4)}$.

\section{Proteína}

A análise de proteinúria com tiras reativas utiliza o princípio do erro protéico dos indicadores. Contrariando a crença geral de que os indicadores produzem determinadas cores em resposta a determinados níveis de $\mathrm{pH}$, certos indicadores mudam de cor devido à presença ou ausência de proteínas, embora o pH do meio permaneça constante $^{(12,14)}$. Em determinado $\mathrm{pH}$, alguns indicadores conferem colorações diversas, dependendo da presença de proteína na solução testada ${ }^{(7)}$. Os indicadores diferem dependendo do fabricante, podendo ser utilizado o azul de tetrabromofenol (Multistix) ou a tetraclorofenoltetrabromossulfoftaleína (Roche).

\section{Urobilinogênio}

A detecção de urobilinogênio na urina através de tiras reativas pode ser feita com o reagente de Ehrlich ( $p$-dimetilaminobenzaldeído), produzindo coloração marrom-avermelhada ${ }^{(7)}$. Esse mesmo reagente é utilizado no controle de qualidade de determinados fármacos, em ensaios de identificação e pureza desses ${ }^{(4)}$. A bula Multistix cita esse reagente como dietilaminobenzaldeído. $\mathrm{O}$ uso de um sal de diazônio derivado de metoxibenzeno é descrito na literatura como mais específico que o reativo de Ehrlich ${ }^{(14)}$. A tira da Roche utiliza uma substância desse grupo.

\section{Limites de deteç̧ão}

O limite mínimo de detecção declarado pelas bulas (Tabela 2) varia dependendo do parâmetro analisado, provavelmente devido às diferenças citadas anteriormente em relação aos reagentes utilizados por cada uma das tiras. Uma diferença importante diz respeito aos valores de sensibilidade citados para hemoglobina, em que a tira Multistix fornece o resultado como concentração de hemoglobina, enquanto a marca Combur relata como eritrócitos $/ \mu \mathrm{l}$, dificultando assim a interpretação, uma vez que os valores de hemoglobina variam entre a população, não necessariamente obtendo o mesmo valor em um determinado número de eritrócitos. Convém relatar que alguns estudos ${ }^{(16)}$ já demonstraram a observação de incompatibilidades entre os valores de sensibilidade citados nas bulas e os realmente encontrados, demonstrando a necessidade de uma revisão das informações reveladas pelos próprios fabricantes.

\section{Intervalos de leitura}

Uma grande disparidade foi observada nos intervalos de leitura citados nas bulas (Tabela 3). Para alguns parâmetros não há diferenças significativas, porém, em determinações como a da glicose, a tira Combur considera a concentração de $300 \mathrm{mg} / \mathrm{dl}$ como positivo (+++), enquanto a marca Multistix coloca uma concentração próxima de $250 \mathrm{mg} / \mathrm{dl}$ com apenas uma cruz (+) de positividade. Sendo assim, em laboratórios que elaboram os laudos da parte química do exame parcial de urina em cruzes, a interpretação do resultado pelo médico fica dificultada, tendo-se em vista a grande variedade de marcas de tiras reativas atualmente disponíveis no mercado.

Nos parâmetros hemoglobina e esterase de leucócitos, os valores de positividade na marca Combur são citados em números, porém na tira Multistix são colocados como atributos qualitativos (baixo, moderado e alto), tornando difícil uma comparação. Na tira Combur resultados de cetonas são dados somente em cruzes, enquanto a Multistix coloca apenas como atributos e concentração. Isso demonstra a necessidade urgente de uma padronização nos exames de urinálise, tanto pelos laboratórios, quanto pelos fabricantes de produtos utilizados na análise da urina. Há atualmente um projeto de norma elaborado pela Comissão de Estudo de Sistema de Referência (CE-36:000.02) da Associação Brasileira de Normas Técnicas/Comitê Brasileiro de Análises Clínicas e Diagnóstico In Vitro: Projeto 36:000.02-003 - Laboratório clínico - Requisitos e recomendações para o exame da urina ${ }^{(1)}$, onde consta que, na realização do exame químico, os testes com tiras reagentes devem ser processados seguindo as instruções do fabricante, não relatando, porém, nenhuma informação referente aos intervalos de leitura utilizados pelas diferentes marcas de tiras.

\section{Potenciais interferências}

As interferências relatadas também apresentam diferenças que podem ser justificadas pelos distintos reagentes empregados pelas tiras, bem como pela escassez de estudos nessa área, principalmente em relação à interferência de medicamentos nos parâmetros avaliados.

A tira Combur apresenta as informaç̧ões da mesma forma nas quatro línguas. A bula da marca Multistix, porém, dispõe as informações de maneira diferente e, em alguns casos, relatando interferências em apenas um dos idiomas, como é o 
caso da esterase de leucócitos, em que se encontra a citação adicional (apenas em espanhol) da possibilidade de o fármaco nitrofurantoína mascarar a reação de cor. As interferências na reação para bilirrubina, em que metabólitos de medicamentos como Pyridium ${ }^{\circledast} \mathrm{e}$ Serenium ${ }^{\circledR}$ podem causar reações falso-positivas, também só são apresentadas em espanhol.

Em relação a essa última informação, merece destaque o fato de as bulas não utilizarem o nome genérico do fármaco em questão. Pyridium ${ }^{\circledR}$ é um nome comercial utilizado para a fenazopiridina nos Estados Unidos ${ }^{(5)}$. Esse fármaco é também comercializado no Brasil em associações de medicamentos, com pelo menos uma dezena de outros nomes comerciais. Em princípio, essas outras especialidades farmacêuticas também poderiam causar interações. Outro exemplo é o Serenium ${ }^{\circledR}$, medicamento citado na bula como potencialmente interferente para bilirrubina e que corresponde a um benzodiazepínico comercializado com diferentes nomes comerciais: Serenium $A D^{\circledR}$, Diepin $A D^{\circledR}$, Mazepam $^{\circledast}$, Nobrix ${ }^{\circledR}$, entre outros ${ }^{(9)}$.

\section{Implicações clínicas e limitações do estudo}

O problema da disparidade nos intervalos de leitura, já relatado, pode dificultar a interpretação dos resultados por parte dos médicos, uma vez que o resultado em cruzes pode corresponder, para diferentes tiras, a concentrações mar- cantemente diferentes do analito. A omissão de relatos de potenciais interferências ou a menção a um nome comercial específico, por outro lado, também pode levar a dificuldades na interpretação de eventuais resultados falso-positivos ou falso-negativos que não sejam reconhecidos como tal.

O presente estudo tem limitações no que diz respeito à grande diversidade de tiras reagentes existentes atualmente no mercado. Analisamos apenas duas das marcas mais tradicionais, mas é provável que a falta de padronização aqui discutida também seja verificada em outras marcas.

\section{Conclusão}

A comparação de duas bulas de tiras reagentes de marcas distintas apontou algumas diferenças nos reativos utilizados, bem como grande diversidade de informações relatadas nas bulas quanto a possíveis interferentes, inclusive com omissão de informações.

Notável diferença foi observada em relação à avaliação semiquantitativa da intensidade da reação e sua expressão em cruzes, por exemplo, nos parâmetros proteína e glicose, o que pode levar a equívocos na interpretação do laudo laboratorial.

As observações registradas reforçam a importância de padronização no exame parcial de urina.

\section{Referências}

I. ASSOCIAÇÃO BRASILEIRA DE NORMAS TÉCNICAS. Laboratório clínico: requisitos e recomendações para o exame de urina, CE- 36.000.02-003. Rio de Janeiro, 2005. 8 p.

2. BALISTRERI, W. F.; REJ, R. Função hepática. In: BURTIS, C. A. ASHWOOD, E. R. Tietz. Fundamentos de química clínica. 4. ed. Rio de Janeiro: Guanabara Koogan, 1998. cap. 31 p. $531-2$

3. BOLODEOKU, J.; DONALDSON, D. Urinalysis in clinical diagnosis. J Clin Pathol, v. 49, n. 8, p. 623-6, 1996.

4. HARTKE, K.; MUTSCHLER, E. DAB 9 - Kommentar. Stuttgart/ Frankfurt:Wissenschaftliche/Govi, 1987. Band I, p.407, 5 I I, 539-540.

5. LACY, C. F. et al. Drug information handbook. 8. ed. Ohio: LexiComp's Clinical Reference Library, 2000-2001. p. 931.

6. LAMMERS, R. L. et al. Comparison of test characteristics of urine dipstick and urinalysis at various test cutoff points. Ann Emerg Med, v. 38, n. 5, p. 505-12, 200 I.

7. LIMA, O.A. et al. Métodos de laboratório aplicados à clínica:Técnica e interpretação. 8. ed. Rio de Janeiro: Guanabara Koogan, 2001. p. 4.1-4.16.
8. LYON, M. E. et al. A preliminary evaluation of the interaction between urine specific gravity and leukocyte esterase using Bayer Multistix ${ }^{\circledR}$ and the Clinitek ${ }^{\circledR}$ 500. Clin Biochem, v. 36 p. 579-82, 2003

9. MOFFAT, A. C.; OSSELTON, M. D.; WIDDOP, B. Clarke's analysis of drugs and poisons: in pharmaceuticals, body fluids and postmortem material. 3 ed., v. 2. London/Chicago: Pharmaceutical Press, 2004. p. 1206.

10. RAVEL, R. Laboratório clínico. Aplicações clínicas dos dados laboratoriais. 6. ed. Rio de Janeiro: Guanabara Koogan, 1997. p. 130-45

II. SACKS, D. B. Glicídeos. In: BURTIS, C. A.; ASHWOOD, E. R Tietz. Fundamentos de química clínica. 4. ed. Rio de Janeiro: Guanabara Koogan, 1998. Cap. 21, p. 352-4.

12. SILVERMAN, L. M.; CHRISTENSON, R. H. Aminoácidos. In: BURTIS, C. A.; ASHWOOD, E. R. Tietz. Fundamentos de química clínica. 4. ed. Rio de Janeiro: Guanabara Koogan, 1998. Cap. 18, p. 269-72.

13. STATLAND, B. E.; WINKEL, P. Preparo de pacientes e amostras para testes laboratoriais. In: HENRY, J. B. Diagnósticos clínicos 
e tratamento por métodos laboratoriais. 19. ed. São Paulo: Manole, 1999. Cap. 9, p. 79-92.

I4.STRASINGER, S. K. Uroanálise e fluidos biológicos. 3. ed. São Paulo: Editora Premier, 1996. p. I-75.

15. SULTANA, R.V. et al. Dipstick urinalysis and the accuracy of the clinical diagnosis of urinary tract infection. J Emerg Med, v. 20, n. I, p. 13-9, 2001.

16.TREITINGER, A. et al. Avaliação de tiras reagentes utilizadas na análise de urina para os parâmetros proteínas, glicose e hemácias. Revista Laes \& Haes, v. I I4, p. 154-68, 1999. 